\title{
Device Space Design for Efficient Scale-Space Edge Detection
}

\author{
B.W. Scotney, S.A. Coleman, M.G. Herron \\ School of Information and Software Engineering, University of Ulster, \\ Coleraine, Northern Ireland \\ \{bw.scotney, sa.coleman. mg.herron\}@ulst.ac.uk
}

\begin{abstract}
We present a new approach to the computation of scalable image derivative operators, based on the finite element method, that addresses the issues of method, efficiency and scale-adaptability. The design procedure is applied to the problem of approximating scalable differential operators within the framework of Schwartz distributions. Within this framework, the finite element approach allows us to define a device space in which scalable image derivative operators are implemented using a combination of piecewise-polynomial and Gaussian basis functions.

Here we illustrate the approach in relation to the problem of scale-space edge detection, in which significant scale-space edge points are identified by maxima of existing edge-strength measures that are based on combinations of scale-normalised derivatives. We partition the image in order to locally identify approximate ranges of scales within which significant edge points may exist, thereby avoiding unnecessary computation of edge-strength measures across the entire range of scales.
\end{abstract}

\section{Introduction}

It is well known that the strength of a feature in an image may depend on the scale at which the appropriate detection operator is applied. It is also the case that many features in images exist significantly (in terms of operator response) over only a limited range of scales, and, moreover, that the most salient scale may vary spatially over the feature. Hence, when designing feature detection operators, it is necessary to consider the requirements for both the systematic development and efficient application of such operators adaptively over appropriately limited scale- and imagedomains.

For scalable image operators, the finite element method offers a framework for systematic development and efficient implementation. In particular, scalable image derivative operators may be approximated within the framework of Schwartz distributions [4]. The general technique may be applied to a range of operators and using a variety of discrete approximations. The issue of scale (see, for example, $[4,5,6])$ is naturally and explicitly embraced in this approach by the introduction into the finite element method of Gaussian test functions that are controlled by a scale parameter [1]. We discuss the efficient implementation of the approach in terms of some well-established mechanisms for implementing the finite element method. It is also shown that the general finite element method naturally overcomes the 'border' problem [7]: image geometries such as corner and boundary operators may be produced with no additional effort. This is particularly important when scale-space techniques are used for problems that may require the use of very large operators. 


\section{Schwartz Distributions}

An image here is represented by a rectangular $n \times n$ array of samples of a continuous function $u(x, y)$ of image intensity on a domain $\Omega$. For the most refined level of finite element discretisation of the image domain $\Omega$, nodes are placed at the pixel centres, and lines joining these form the edges of elements (see Fig. 1).

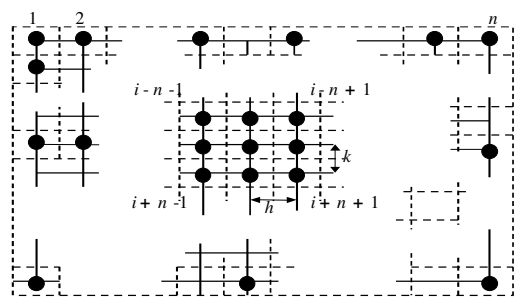

Fig. 1. Regular rectangular array of pixels and nodes for finite element discretisation

In Fig. 1 a global numbering scheme for the nodes is depicted. The dotted lines show the natural pixel edges.

In previous work $[8,9]$ we have formulated image operators that correspond to weak forms of operators in the finite element method [2]. Operators used for smoothing may be based simply on a weak form of the image function, for which it is assumed that the image function $u \equiv u(x, y)$ belongs to the Hilbert space $H^{0}(\Omega)$; that is, the integral $\int_{\Omega} u^{2} d \Omega$ is finite. Edge detection and enhancement operators are often based on first or second derivative approximations, for which it is necessary that the image function $u \equiv u(x, y)$ is constrained to belong to the Hilbert space $H^{1}(\Omega)$; i.e. the integral $\int_{\Omega}\left(|\underline{\nabla} u|^{2}+u^{2}\right) d \Omega$ is finite, where $\underline{\nabla} u$ is the vector $(\partial u / \partial x, \partial u / \partial y)^{T}$.

We now embrace a more general, and less restrictive, approach offered by Schwartz distribution theory (see [4]). For the image function $u \equiv u(x, y)$ we may obtain a distribution $F_{u}[v] \equiv \int_{\Omega} u v d \Omega$ for some test function $v$. Derivatives of order $\alpha$ are then defined by $\left(\nabla_{\alpha} F_{u}\right)[v] \equiv F_{u}\left[(-1)^{|\alpha|} \nabla_{\alpha} v\right]$. Hence a first or second order directional derivative may be defined by (respectively)

$$
E(u)=-\int_{\Omega} \underline{u b} \cdot \underline{\nabla} v d \Omega \text { and } Z(u)=\int_{\Omega} u \underline{\nabla} \cdot(\mathrm{B} \underline{\nabla} v) d \Omega
$$

Here $\mathbf{B}=\underline{b} \underline{b}^{\mathrm{T}}$ and $\underline{b}=(\cos \theta, \sin \theta)$ is the unit direction vector. Zero-crossing methods are often based on the isotropic form of the second order derivative, namely the Laplacian. In this formulation, this is equivalent to the general form in which the matrix $\mathbf{B}$ is the identity matrix $\mathbf{I}$. 


\section{Finite Element Formulation}

In the finite element method a finite-dimensional space $S^{h}$ is used for function approximation. We use this idea to implement an "image space" $S^{h}$. In the image space context, the superscript $h$ corresponds to the inter-pixel distance in the image array (see Fig. 1). A finite element mesh is constructed using rectangular elements, as in Fig. 1, in which a global numbering scheme $1, \ldots, N$ is employed. A basis for $S^{h}$ may be formed by associating with each node $i$ a basis function $\phi_{i}(x, y)$ which has the properties

$$
\phi_{i}\left(x_{j}, y_{j}\right)= \begin{cases}1 & \text { if } i=j \\ 0 & \text { if } i \neq j\end{cases}
$$

where $\left(x_{j}, y_{j}\right)$ are the co-ordinates of the nodal point $j . \phi_{i}(x, y)$ is thus a "tentshaped" function with support restricted to a neighbourhood, centred on node $i$, consisting of only those elements that have node $i$ as a vertex. We then approximately represent the image $u$ by a function $U(x, y)=\sum_{j=1}^{N} U_{j} \phi_{j}(x, y)$ in which the parameters $\left\{U_{1}, \ldots, U_{N}\right\}$ are mapped from the sampled image intensity values. The approximate image representation is therefore a simple function (typically a low order polynomial) on each element and has the sampled intensity value $U_{j}$ at node $j$.

Our previous work has been based on a formulation analogous to the Galerkin finite element method [9]. However, since we now focus on the development of operators that can explicitly embrace the concept of scale, an alternative approach is preferred in which a finite-dimensional test space $T_{\sigma}^{h}$ other than $S^{h}$ is employed; the test space, or "device space", $T_{\sigma}^{h}$ explicitly embodies a scale parameter $\sigma$. This generalisation allows sets of test functions $\psi_{i}^{\sigma}(x, y), i=1, \ldots, N$, to be used when defining scale-space derivatives; for first and second order operators respectively, this provides the functionals

$$
E_{i}^{\sigma}(U)=-\int_{\Omega} U \underline{b}_{i} \cdot \underline{\nabla} \psi_{i}^{\sigma} d \Omega_{i} \text { and } Z_{i}^{\sigma}(U)=\int_{\Omega} U \underline{\nabla} \cdot\left(\mathbf{B}_{i} \underline{\nabla} \psi_{i}^{\sigma}\right) d \Omega
$$

The scale parameter $\sigma$ may now be used to explicitly control the scale of the operator, as illustrated in the example implementation in Section 4.

\section{Example Implementation}

We illustrate the approach applied to first derivative approximation using a regular bilinear rectangular discretisation formed from a set of rectangular elements such as that illustrated in Fig. 1. We construct a set of basis functions $\phi_{i}(x, y), i=1, \ldots, N$, so that the $N$-dimensional image subspace $S^{h}$ comprises of functions that are piecewise bilinear. 
For the test space $T_{\sigma}^{h}$ a set of Gaussian basis functions is used that explicitly embodies the scale parameter $\sigma$. Sets of Gaussian test functions $\psi_{i}^{\sigma}(x, y), i=1, \ldots, N$ of the form

$$
\psi_{i}^{\sigma}(x, y)=\frac{1}{2 \pi \sigma^{2}} e^{-\left(\frac{\left(x-x_{i}\right)^{2}+\left(y-y_{i}\right)^{2}}{2 \sigma^{2}}\right)}
$$

are constructed, each restricted to have support over a neighbourhood $\Omega_{i}^{\sigma}$ centred on the node $i$ at $\left(x_{i}, y_{i}\right)$. The size of the neighbourhood $\Omega_{i}^{\sigma}$ to which the support of $\psi_{i}^{\sigma}(x, y)$ is restricted is also explicitly related to the scale parameter $\sigma$ [3]. The Gaussian is restricted so that the neighbourhood $\Omega_{i}^{\sigma}$ is a $2 W_{\sigma} \times 2 W_{\sigma}$ block of rectangular elements (where $W_{\sigma}$ is an integer) with the nodal position $\left(x_{i}, y_{i}\right)$ at the centre of the block. Then the relationship $1.96 \sigma=W_{\sigma}$ ensures that along both the vertical and horizontal co-ordinate directions through the nodal point $\left(x_{i}, y_{i}\right), 95 \%$ of the cross-section of the Gaussian is contained within $\Omega_{i}^{\sigma}$.

On a neighbourhood $\Omega_{i}^{\sigma}$ we consider a locally constant unit vector $\underline{b}_{i}=\left(b_{i 1}, b_{i 2}\right)^{T}$. Substituting the image representation $U(x, y)=\sum_{j=1}^{N} U_{j} \phi_{j}(x, y)$ into the derivative $E_{i}^{\sigma}(U)=-\int_{\Omega} U \underline{b}_{i} \cdot \underline{\nabla} \psi_{i}^{\sigma} d \Omega_{i}$ gives

$$
E_{i}^{\sigma}(U)=-b_{1 i} \sum_{j=1}^{N} K_{i j}^{\sigma} U_{j}-b_{2 i} \sum_{j=1}^{N} L_{i j}^{\sigma} U_{j},
$$

where $K_{i j}^{\sigma}$ and $L_{i j}^{\sigma}$ are respectively entries in $N \times N$ global matrices $K^{\sigma}$ and $L^{\sigma}$ given by

$$
K_{i j}^{\sigma}=\int_{\Omega_{i}^{\sigma}} \frac{\partial \psi_{i}^{\sigma}}{\partial x} \phi_{j} d x d y \text { and } L_{i j}^{\sigma}=\int_{\Omega_{i}^{\sigma}} \frac{\partial \psi_{i}^{\sigma}}{\partial y} \phi_{j} d x d y, i, j=1, . ., N .
$$

These integrals need be computed only over the neighbourhood $\Omega_{i}^{\sigma}$, rather than the entire image domain $\Omega$, since $\psi_{i}^{\sigma}$ has support restricted to $\Omega_{i}^{\sigma}$.

\subsection{Element-by-Element Computation}

Each neighbourhood $\Omega_{i}^{\sigma}$ is composed of a set $S_{i}^{\sigma}$ of elements. In our example implementation $S_{i}^{\sigma}$ is the $2 W_{\sigma} \times 2 W_{\sigma}$ block of elements having nodal point $\left(x_{i}, y_{i}\right)$ at its centre. We may thus write $K_{i j}^{\sigma}$ as a summation $K_{i j}^{\sigma}=\sum_{\left\{m e_{m} \in S_{i} S^{\sigma}\right\}} k_{i j}^{m, \sigma}$ where $k_{i j}^{m, \sigma}$ is the element integral $k_{i j}^{m, \sigma}=\int_{e_{m}} \frac{\partial \phi_{j}}{\partial x} \psi_{i}^{\sigma} d x d y$. 
In principle, each $E_{i}^{\sigma}(U)$ may be computed by calculating $k_{i j}^{m, \sigma}$ and $l_{i j}^{m, \sigma}$ for each element $e_{m}$ in $S_{i}^{\sigma}$ and then assembling these values to yield $K_{i j}^{\sigma}$ and $L_{i j}^{\sigma}$ respectively. In practice, much less computation than this is required. To construct $E_{i}^{\sigma}(U)$ only one quarter of the operator is computed, and via reflective symmetry in the horizontal and vertical axes the complete operator is generated. Consider the situation in Fig. 2 where the Gaussian basis function is restricted to the $4 \times 4$ block of elements (i.e. $W^{\sigma}=2$ ). We need to compute $k_{i j}^{m, \sigma}$ and $l_{i j}^{m, \sigma}$ only for $m=a_{1}, b_{1}, c_{1}, d_{1}$, representing just one quarter of the whole support $\Omega_{i}^{\sigma}$ of the operator $E_{i}^{\sigma}$.

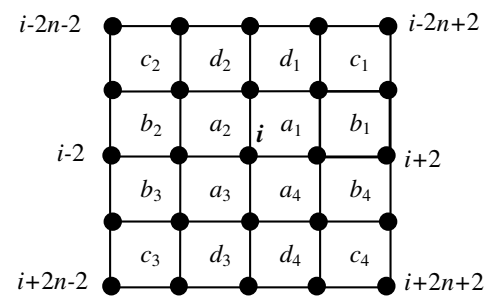

Fig. 2. Elements within neighbourhood $\Omega_{i}^{\sigma}$ for $\mathrm{W}^{\sigma}=2$

For each of the elements $e_{m}, m=a_{1}, b_{1}, c_{1}$ and $d_{1}$, a pair of $2 \times 2$ element operators, $k_{i}^{m, \sigma}$ and $l_{i}^{m, \sigma}$, is generated. For example, the $x$-directional element operator over element $a_{1}$ is given by:

$$
k_{i}^{a_{1}, \sigma}=\left[\begin{array}{cc}
k_{i, i-n}^{a_{1}, \sigma} & k_{i, i, n+1}^{a_{1}, \sigma} \\
k_{i, i}^{a_{1}, \sigma} & k_{i, i+1}^{a_{1}, \sigma}
\end{array}\right] \text { where } k_{i j}^{a_{1}, \sigma}=\int_{a_{1}} \frac{\partial \phi_{j}}{\partial x} \psi_{i}^{\sigma} d x d y .
$$

If the element sizes are normalised, i.e. $h=k=1$, this can readily be calculated and found to be:

$$
a_{1}=\left[\begin{array}{cc}
\alpha_{4} & \alpha_{3} \\
\alpha_{1} & \alpha_{2}
\end{array}\right]=\left[\begin{array}{ll}
-0.025178 & 0.025178 \\
-0.021469 & 0.021469
\end{array}\right]
$$

In practice, integration over an element is done numerically using a low-order Gauss quadrature rule, typically requiring just four evaluations of the integrand (e.g. [2]). Similarly, we can compute:

$$
\begin{aligned}
& b_{1}=\left[\begin{array}{ll}
\beta_{4} & \beta_{3} \\
\beta_{1} & \beta_{2}
\end{array}\right]=\left[\begin{array}{ll}
-0.061061 & 0.061061 \\
-0.052064 & 0 .
\end{array}\right], \quad c_{1}=\left[\begin{array}{ll}
\gamma_{4} & \gamma_{3} \\
\gamma_{1} & \gamma_{2}
\end{array}\right]=\left[\begin{array}{ll}
-0.011802 & 0.011802 \\
-0.007433 & 0.007433
\end{array}\right], \\
& \text { and } d_{1}=\left[\begin{array}{ll}
\delta_{4} & \delta_{3} \\
\delta_{1} & \delta_{2}
\end{array}\right]=\left[\begin{array}{ll}
-0.028621 & 0.028621 \\
-0.018025 & 0.018025
\end{array}\right]
\end{aligned}
$$


We can generate operators of any size by this means, only ever requiring four precomputed values of the basis function derivative $D X[\cdot][g]=\left.\frac{\partial \phi}{\partial x}\right|_{\left(x_{g}, y_{g}\right)}$ evaluated at four Gauss quadrature points in a representative element. The four values of $D X[\cdot][g]$ for each node in any element can be obtained by permutation of these four pre-computed values. Further, since the corresponding four values of $D Y[\cdot][g]$ required for the computation of $l_{i}^{m, \sigma}$ are permutations of the four values of $D X[\cdot][g]$, it is not necessary to explicitly compute $l_{i}^{m, \sigma}$; the operator $L_{i j}^{\sigma}$ is merely a rotation of the operator $K_{i j}^{\sigma}$. The general procedure for computing the contributions required for $K_{i j}^{\sigma}$ is shown below.

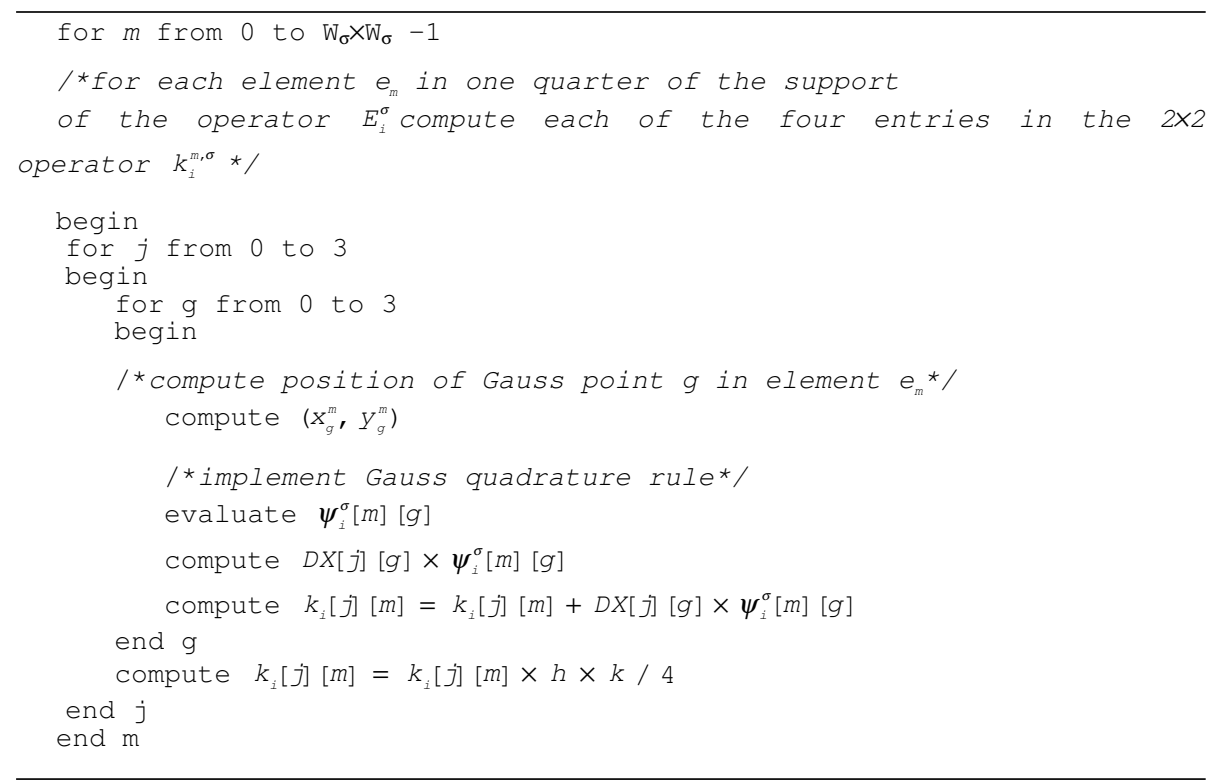

The assembly process to construct $E_{i}^{\sigma}(U)$ from the element matrices is described in Section 4.2.

\subsection{Finite Element Assembly}

The next stage of the finite element method consists of assembling the element matrices $k_{i}^{m, \sigma}$ and $l_{i}^{m, \sigma}$, for each element $m=1, \ldots, M$, into the global matrices $K^{\sigma}$ and $L^{\sigma}$ respectively. This is accomplished by distributing each entry in the element matrices into the corresponding row and column of the global matrices using the local to global node numbering mapping routinely stored in the finite element method. 


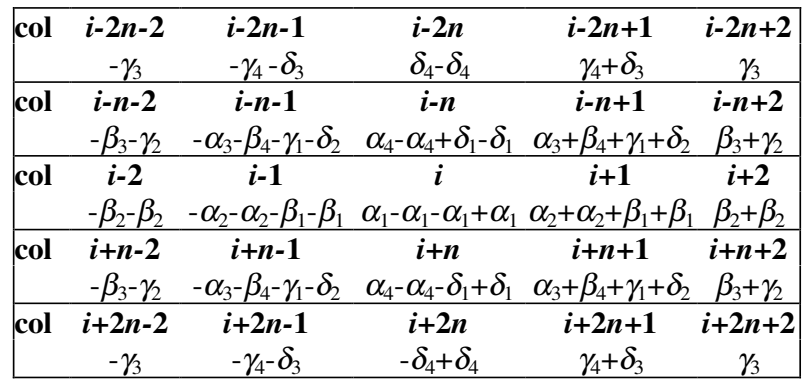

Fig. 3. Element assembly to form row $i$ of matrix $\boldsymbol{K} \sigma$

Such a procedure is illustrated in Fig. 3 for the distribution of the values of the 16 element matrices $k_{i}^{m, \sigma}$ corresponding to the 16 elements depicted in Fig. 2. We see that 25 values of the global matrix $K^{\sigma}$ are augmented in the assembly procedure for these 16 elements. Since the neighbourhood $\Omega_{i}^{\sigma}$ is centred on node $i$, these 25 values are all in row $i$ of $K^{\sigma}$ and in the columns of $K^{\sigma}$ indicated in Fig. 2. Hence assembled in row $i$ of the global matrix $K^{\sigma}$ we have the $5 \times 5$ spatial operator centred on node $i$ :

\begin{tabular}{|ccccc|}
\hline$-\gamma_{3}$ & $-\gamma_{4}-\delta_{3}$ & 0 & $\gamma_{4}+\delta_{3}$ & $\gamma_{3}$ \\
$-\beta_{3}-\gamma_{2}$ & $-\alpha_{3}-\beta_{4}-\gamma_{1}-\delta_{2}$ & 0 & $\alpha_{3}+\beta_{4}+\gamma_{1}+\delta_{2}$ & $\beta_{3}+\gamma_{2}$ \\
$-2 \beta_{2}$ & $-2 \alpha_{2}-2 \beta_{1}$ & 0 & $2 \alpha_{2}+2 \beta_{1}$ & $2 \beta_{2}$ \\
$-\beta_{3}-\gamma_{2}$ & $-\alpha_{3}-\beta_{4}-\gamma_{1}-\delta_{2}$ & 0 & $\alpha_{3}+\beta_{4}+\gamma_{1}+\delta_{2}$ & $\beta_{3}+\gamma_{2}$ \\
$-\gamma_{3}$ & $-\gamma_{4}-\delta_{3}$ & 0 & $\gamma_{4}+\delta_{3}$ & $\gamma_{3}$ \\
\hline
\end{tabular}

Assembly of all of the element matrices into $K^{\sigma}$ thus yields a sparsely populated and highly structured $N \times N$ block matrix composed of $n \times n$ sub-matrices. Each submatrix is itself sparse, having non-zero values in only 5 positions in each row: the diagonal, and the first two upper and lower diagonals.

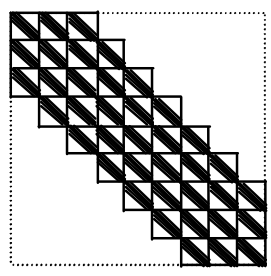

Fig. 4. The general banded block structure of $\boldsymbol{K}^{\sigma}$

Fig. 4 shows the general structure of $K^{\sigma}$; zero-values have been suppressed.

Each row of matrix $K^{\sigma}$ and of matrix $L^{\sigma}$ represents an operator on the vector $\mathbf{U}=\left\{U_{j}\right\}, j=1, \ldots, N$, by which the scale-space derivative approximations $E_{i}^{\sigma}, i=1, \ldots, N$ are computed. As an integral part of this process, appropriate operators are generated both for image corners and image borders as well as the internal regions of the image. Hence the "border problem", particularly significant for large-scale operators, is naturally accommodated. 
We thus obtain the vector $E^{\sigma}=\left\{E_{i}^{\sigma}\right\}$, for $i=1, \ldots, N$, of weak first derivative approximations by using the operator $\left(B_{1}^{\sigma} K^{\sigma}+B_{2}^{\sigma} L^{\sigma}\right)$ on the vector $\mathbf{U}$ of image intensity values, namely,

$$
E^{\sigma}=\left(B_{1}^{\sigma} K^{\sigma}+B_{2}^{\sigma} L^{\sigma}\right) \mathbf{U}
$$

where $B_{1}^{\sigma}$ and $B_{2}^{\sigma}$ are diagonal matrices of respectively the locally constant direction components $b_{i 1}$ and $b_{i 2}$ on each neighbourhood $\Omega_{i}^{\sigma}$ as described in Section 3 .

\section{Scale-Space Edge Detection}

As a fundamental application of the technique for adaptive scale-space derivative approximation developed above, we choose the problem of scale-space edge detection. For this we follow closely the work on edge detection with automatic scale selection developed in [6]. In [6], significant scale-space edge points are identified by maxima of specified edge-strength measures that are located by zero-crossings of two functions $Z_{1}(L)$ and $Z_{2}(L)$ of the scale-space image $L(x, y, t)$, where $t$ is the scale parameter. The procedure used in [6] is a pre-processing phase to tracking the zerocrossing surfaces and identifying, by use of a line integral measure, the most significant scale-space edges in the image. In [6] it is illustrated that this approach can be very successful in both identifying the most significant edges and demonstrating how the most salient scale varies along an edge.

We now illustrate an alternative approach to the pre-processing phase in [6] based on application of the adaptive scale-space derivative approximation technique developed in Sections 2 to 4. Advantages of our approach are that we exploit the fact that the image features exist significantly over a limited range of scales to avoid the computation of global scale-space images over the extensive range of scales used in [6]; moreover, our adaptive normalised scale-space derivatives naturally and systematically combine the Gaussian convolution smoothing and discrete derivative approximation steps that are carried out separately in [6], thus avoiding the use of ad hoc finite difference approximations.

As an initial step we partition the image into an array of regular rectangular subregions, $R_{j}, j=1, \ldots, J$, within each of which we compute the image variance $\sigma_{j}$. The results of numerical experimentation have established that $\sigma_{j}$ may be used as an indicator of the appropriate device space to be used for the computation of the scalespace derivatives within $R_{j}$ required for $Z_{1}(L)$ and $Z_{2}(L)$. Using the experimentally derived relationship $\sigma_{j}^{0}=1 / \log _{10} \sigma_{j}$, we thus compute the operator scale $\sigma_{j}^{0}$ at which the device space response is approximately maximal. Hence a "key-scale" $t_{j}^{0}$ is established for each sub-region $R_{j}$. The operator size corresponding to $t_{j}^{0}$ is 
automatically derived using the procedure described in Section 4, and Fig. 5 shows an image together with these "key scales" computed for 64 image sub-regions. Here, "1" indicates $3 \times 3$, " 2 " indicates $5 \times 5$, etc., and it can be clearly seen that the smaller scales occur in regions representing the detail image objects, whilst larger scales are employed in the image background.

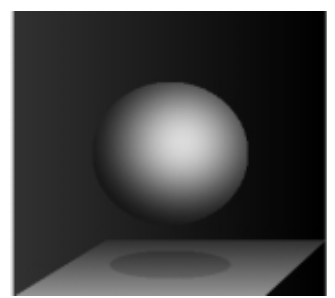

(a) $256 \times 256$ Original image

\begin{tabular}{|l|l|l|l|l|l|l|l|}
\hline 7 & 8 & 8 & 7 & 7 & 8 & 8 & 8 \\
\hline 788 & 7 & & & 78 & 8 & & \\
\hline 782 & 1 & & & 11 & 8 & & \\
\hline 781 & 1 & & & 21 & 8 & & \\
\hline 781 & 1 & & & 1188 & & \\
\hline 78 & 1 & & & 11 & 8 & & \\
\hline 722 & 2 & & & 211 & 1 & & \\
\hline 1 & 1 & 1 & 1 & 1 & 1 & 1 & 1 \\
\hline
\end{tabular}

(b) Image sub-regions with corresponding operator scales

Fig. 5. Illustration of adaptive filters

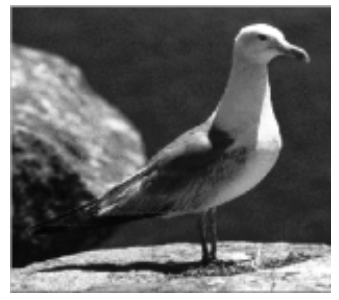

(a) $256 \times 256$ Original image

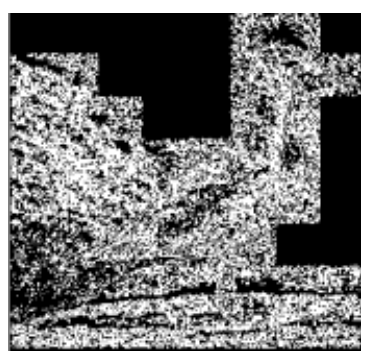

(c) all scale-space edge points from the adaptive method

\begin{tabular}{|l|l|l|l|l|l|l|l|}
\hline 2 & 2 & 2 & 3 & 2 & 1 & 1 & 2 \\
\hline 113 & 2 & & & 211 & 1 & & \\
\hline 111 & 2 & & & 21 & 2 & & \\
\hline 111 & 1 & & & 11 & 2 & & \\
\hline 111 & 1 & & & 11 & 2 & & \\
\hline 211 & 1 & & & 112 & 2 & & \\
\hline 211 & 1 & & & 11 & 1 & & \\
\hline 111 & 1 & & & 111 & 1 & & \\
\hline
\end{tabular}

(b) Image sub-regions with corresponding operator scales

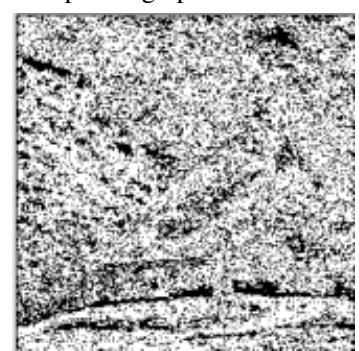

(d) All scale-space edges from the method in [6]

Fig. 6.Scale-Space representations

Fig. 6 (a) and (b) show another image and corresponding grid of sub-regional keyscales. Again the use of smaller scales for the image detail and larger scales for the background is clearly evident. Once a regional key-scale $t_{j}^{0}$ has been computed for each $R_{j}$, the measures $Z_{1}(L)$ and $Z_{2}(L)$ are computed over $R_{j}$ for a small number of scales above and below $t_{j}^{0}$; in this case we have chosen five scales: $t_{j}^{-2}, t_{j}^{-1}, t_{j}^{0}, t_{j}^{1}, t_{j}^{2}$ centred on $t_{j}^{0}$. The inter-spatial distance has been chosen to be the 
same as that used in [6]; note, however, that we are not restricted to aligning the scales between sub-regions, as each key-scale $t_{j}^{0}$ is selected independently. In Fig. 6(c) and (d) respectively are shown all scale-space edge points identified by the method proposed in this paper and by the method described in [6]. Whilst both approaches provide suitable output for scale-space edge tracking by the method used in [6], we note that the scale-space edge map projection generated by our approach is very much sparser, representing a significant reduction in computational effort.

\section{Conclusion}

A key concept in the finite element method is the notion of a standard element. This concept enables convenient and systematic computation to be performed even in complex situations. Because of the sparse banded characteristic of the global matrices produced, efficiency extends to storage as well as computation. We have shown that the concept of scale for operators can be explicitly and efficiently implemented within a finite element framework. In particular, using Schwartz distributions, we have presented a new approach to scalable image derivative operators that addresses the issues of method, efficiency and scale-adaptability. We have illustrated that the approach is well-suited to the fundamental problem of scale-space edge detection: our adaptive normalised scale-space derivatives naturally and systematically combine Gaussian convolution smoothing and discrete derivative approximation.

\section{References}

[1] Babaud,J., Witkin, A.P., Baudin, M., and Duda, R.O., "Uniqueness of the Gaussian Kernel for Scale-Space Filtering" IEEE Trans. on PAMI, Vol. PAMI-8(1), 1986, 26-33.

[2] Becker, E.B., Carey, G.F., and Oden, J.T., Finite Elements: An Introduction, Prentice Hall, London, 1981.

[3] Davies,E.R., "Design of Optimal Gaussian Operators in Small Neighbourhoods" Image and Vision Computing, Vol. 5(3), 1987, 199-205.

[4] Florack, L., Image Structure, Computational Imaging and Vision, Vol. 10, Kluwer Academic Publishers, 1997.

[5] Lindeberg, T., Scale-Space Theory in Computer Vision, Kluwer Academic Publishers, 1994.

[6] Lindeberg, T., "Edge Detection and Ridge Detection with Automatic Scale Selection", International Journal of Computer Vision, Vol. 30(2), 117-154, 1998.

[7] Prewitt, J.M.S., "Object Enhancement and Extraction", Picture Processing \& Psychopictorics, 1970, 75-149.

[8] Scotney, B.W., Coleman, S.A., Herron, M.G., "A Systematic Design Procedure for Scalable Near-Circular Gaussian Operators.", Proceedings of the IEEE Int. Conf. On Image Processing(ICIP 2001), Greece, 844-847, 2001.

[9] Scotney, B.W., Herron, M.G., "Systematic and Efficient Construction of Neighbourhood Operators on Irregular and Adaptive Grids." Third Irish Machine Vision and Image Processing Conference (IMVIP'99), Dublin, 204-218, 1999. 